\title{
HOW HEALTH TECHNOLOGY ASSESSMENT AGENCIES ADDRESS THE ISSUE OF UNPUBLISHED DATA
}

\author{
Julia Kreis \\ Department of Health Care Management, Berlin University of Technology \\ Institute for Quality and Efficiency in Health Care
}

\author{
Dimitra Panteli, Reinhard Busse \\ Department of Health Care Management, Berlin University of Technology
}

Objectives: Reporting bias potentially threatens the validity of results in health technology assessment (HTA) reports. Our study aimed to explore policies and practices of HTA agencies regarding strategies to include previously unpublished data in their assessments, focusing on requests to industry for unpublished data.

Methods: We included international HTA agencies with publicly available methods papers as well as HTA reports. From the methods papers and recent reports we extracted information on requests to industry and on searches in trial registries, regulatory authority Web sites and for conference abstracts.

Results: Eighteen HTA agencies and seventy-three reports were included. Agencies' methods papers showed variability regarding requests to industry (requests are routinely carried out in seven cases, not mentioned in six, at the discretion of HTA authors in three, and based on manufacturer applications in two), which were reflected in the reports investigated. As reporting of requests was limited, it often remained unclear whether unpublished data had been received. Searches in trial registries, at regulatory authorities or for conference abstracts are described as a routine or optional part of the search strategy in the methods papers of 9, 11, and 8 included agencies, respectively. A total of 52 percent, 39 percent, and 16 percent of reports described searches in trial registries, at regulatory agencies, and hand searching of conference proceedings.

Conclusion: International HTA agencies currently differ considerably in their efforts to address the issue of unpublished data. Requests to industry may constitute one strategy to access and include unpublished data, while agencies can learn from each other concerning successful practice.

Keywords: Technology assessment, Biomedical (MeSH), Publication bias (MeSH), Review literature as topic (MeSH)

\section{Evidence on Reporting Bias}

Over the last few decades, evidence has been accumulating on the existence of reporting biases in the medical literature which "arise when the dissemination of research findings is influenced by the nature and direction of results" (p. 298 (1)). A recent overview found examples of reporting bias in 50 different interventions and procedures in the fields of pharmacology, surgery, diagnostics, and prevention, thus demonstrating that this phenomenon poses a major problem in the assessment of health care interventions (2). The best known form of reporting bias is publication bias, defined as "the publication or nonpublication of research findings, depending on the nature and direction of the results" (p. 298 (1)). However, even in published studies, subsets of the original outcomes or analyses may be selectively reported (outcome or analysis reporting bias) (3).

\footnotetext{
The authors thank Kay Dickersin, Milo Puhan, and Annette Zentner for their contributions during the design phase of the study; Elke Hausner and Natalie McGauran for their comments on a draft version of this paper; and Natalie McGauran for editorial support. The views expressed in this article are those of the authors and do not necessarily represent those of the Institute for Quality and Efficiency in Health Care or the University of Technology, Berlin.
}

\section{Challenge for HTA Agencies}

Reporting bias tends to overestimate benefits and underestimate harms of health care interventions, thus leading to potentially biased conclusions in systematic reviews and health technology assessment (HTA) reports $(2 ; 4)$. This was illustrated by a recent assessment of reboxetine by the German HTA agency IQWiG (Institute for Quality and Efficiency in Health Care): the inclusion of data from ten trials, which had previously been unpublished or incompletely published and were provided by the manufacturer only after considerable public pressure, had a significant impact on the assessment's results. IQWiG's conclusion that reboxetine is "overall an ineffective and potentially harmful antidepressant" (p. 8 (5)) stood in contrast to the findings of several earlier systematic assessments that did not combine all of the existing evidence from clinical trials (6), and ultimately led to the exclusion of reboxetine from reimbursement in Germany.

\section{Data Accessible to HTA Agencies}

Legislation requires manufacturers to grant regulatory authorities such as the U.S. Food and Drug Administration (FDA) comprehensive access to both published and unpublished data for the approval process (e.g., (7)). However, manufacturers are not generally obliged to provide all of the relevant data to HTA 
agencies for post-approval evaluations (8). Health policy decisions, for example, on the reimbursement of drugs, are therefore often based mainly on publicly available information with the potential insufficiencies described above (8-10).

One way of overcoming the selective or nonpublication of studies is their registration in public databases: in 2004, the International Committee of Medical Journal Editors specified that registration in a public trial registry was a prerequisite for a study's publication (11). In response to public concerns about reporting biases and to legal proceedings (the paroxetine case (12)), several individual pharmaceutical companies, as well as industry associations such as the Pharmaceutical Research and Manufacturers of America (PhRMA), installed their own registries. In 2007, the FDA Amendments Act made registration and public posting of all applicable clinical trials in the registry ClinicalTrials.gov compulsory for the United States (13). In Europe, the publicly accessible EU Clinical Trials Register was launched only recently (March 2011) and contains information on phase $2-4$ adult clinical trials and any pediatric clinical trial carried out in the European Union from 2004 onward (14). However, existing regulations for trial registration have several loopholes, particularly as older drugs, although widely used in clinical practice, are not covered $(8 ; 15)$.

Other strategies used to identify potentially relevant unpublished studies or data include searching for regulatory authority Web sites as well as conference abstracts, as approximately half of trials reported in conference abstracts are never fully published, and publication is associated with positive results (16). However, it has been argued that publicly available sources do not necessarily contain all relevant clinical trial results for a given healthcare intervention, and that access to these results is still insufficient (13).

As publication is not a dichotomous event, but rather a continuum (17), the distinction between "published" and "unpublished" is not always clear cut (2). In our study, we use the term "published" to refer to articles published in peer-reviewed journals and the term "unpublished" to refer both to publicly inaccessible data owned by industry or study authors and to "gray literature," that is, studies not published in peer-reviewed journals but nevertheless publicly available in sources such as trial registries, regulatory authorities or published as conference abstracts.

\section{Aim of Our Study}

To the best of our knowledge, no detailed analysis is currently available as to how international HTA agencies address the challenge of unpublished data. On the basis of an analysis of publicly available methods papers and HTA reports, the aim of our study was, therefore, to provide an overview of current policies and practices with regard to searching for, including and reporting of unpublished data. Our primary interest was to explore whether and how HTA agencies carry out requests to industry to include data in their assessments that are not publicly available at all. We also explored to what extent alternative strategies are pursued to identify grey literature from trial registries, regulatory authorities and conference abstracts.

\section{METHODS}

\section{Eligible HTA Agencies and Documents}

We analyzed two different data sources: "methods papers," that is, documents describing the HTA organizations' methods for assessing the effectiveness of interventions, and "HTA reports," that is, full assessments of a health technology or focused assessments of drugs for reimbursement and pricing decisions. We aimed for a broad sample to capture current practice in the HTA community and therefore identified international HTA agencies from four different sources: (a) members of the International Network of Agencies for Health Technology Assessment (INAHTA) as of December 2010, (b) partners in the European Network for Health Technology Assessment (EUnetHTA) project as of December 2010, (c) nonprofit members in Health Technology Assessment international (HTAi) as of May 2011, and (d) HTA agencies included in comparative analyses published in the International Journal of Technology Assessment in Health Care in the years 2009 and 2010. Eligible agencies were those that produced HTA reports and for which we were able to identify both eligible methods papers and HTA reports in English, German, or French (for inclusion and exclusion criteria see Supplementary Table 1: http://dx.doi.org/10.1017/S026646231300072X).

\section{Data Collection, Extraction, and Analysis}

We searched all Web sites of potentially eligible agencies at the beginning of 2011, both to check whether they are directly involved in HTA by either commissioning or conducting assessments and to identify methods papers as well as HTA reports. We contacted agencies between February and May 2011 to verify the information obtained online and confirm the completeness and currentness of the documents retrieved. We did not contact agencies where the Web site clearly indicated that they are not involved in HTA production or where insufficient information in English, French, or German was available.

Methods Papers. We included methods papers developed by the respective agencies that were publicly available and described methods for assessing the effectiveness (i.e., not only costeffectiveness) of drug or nondrug therapeutic interventions. We included more than one methods paper if they had different scopes, yet only those for which eligible reports were also publicly available. If further documents explicitly addressing the inclusion and handling of unpublished data were available, they were also included in the analysis. 
Kreis et al.

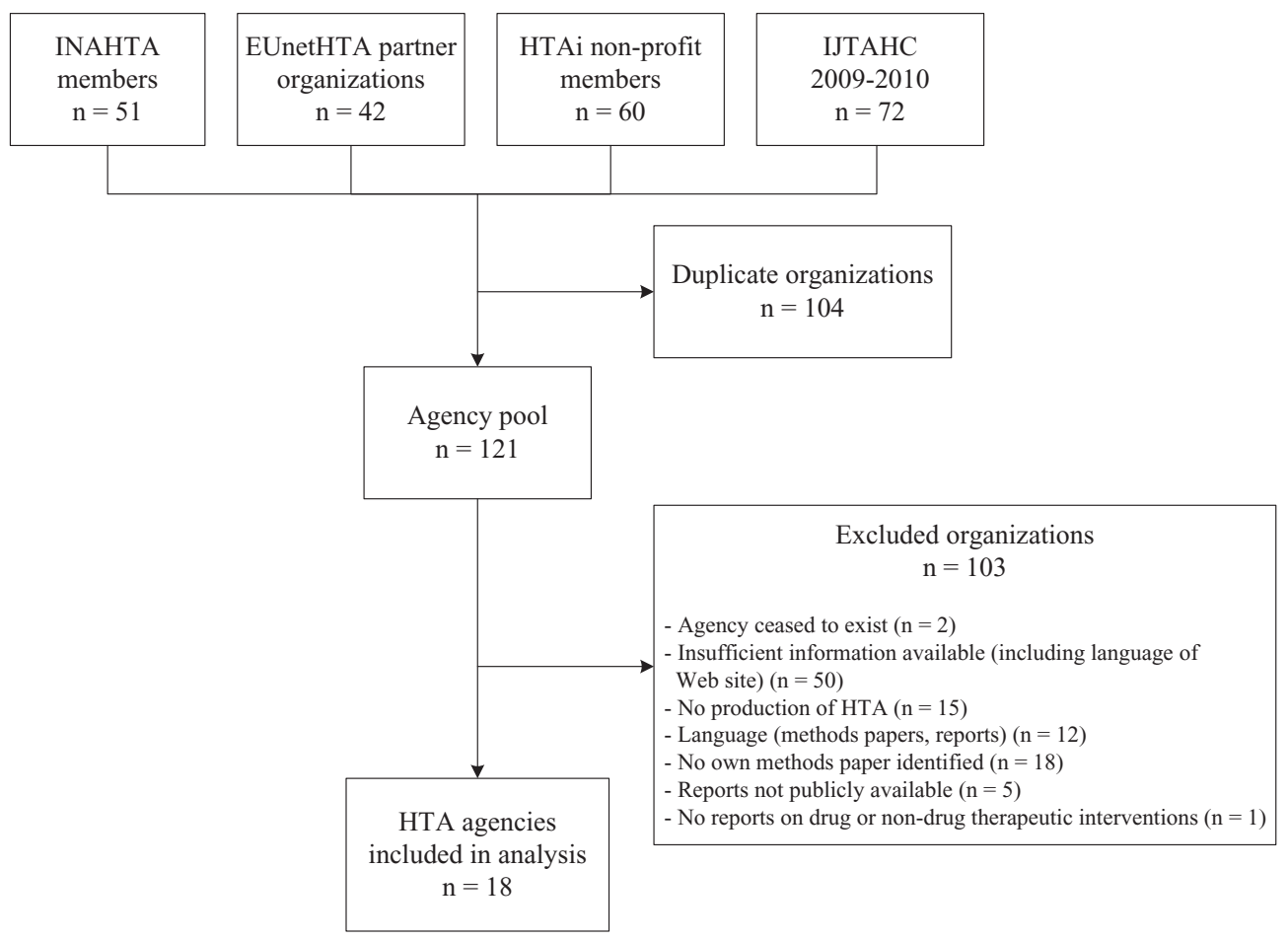

Figure 1. Flow chart of selection of HTA agencies (May 2011).

HTA Reports. We included HTA reports that were publicly available in full (i.e., not only in summary) and reported a systematic evaluation (i.e., based on a search for literature in at least two sources) of the effectiveness of drug or nondrug therapeutic interventions from January 2006 onward. We excluded reports that (i) were commissioned by another agency that was also included in our sample, (ii) were based solely on secondary publications (e.g., "reviews of reviews" that did not include primary studies), or (iii) were draft versions. We included the three most recent reports on both drugs and nondrug therapeutic interventions or fewer if less than three were available.

To reduce the heterogeneity between reports and due to limited resources, we did not examine documents (neither methods papers nor HTA reports) reporting the evaluation of diagnostic procedures or population-based preventive interventions. In addition, for agencies producing different types of documents, we restricted our analysis to one type (e.g., to multiple technology assessments for NICE documents) to reduce the complexity of the analysis.

We developed and pilot-tested sheets to extract information from methods papers and HTA reports. Information was extracted by one person (JK) and checked by a second (DP) for the categories depicted in Supplementary Table 2, which can be viewed online at http://dx.doi.org/ $10.1017 /$ S026646231300072X. Disagreements were solved by discussion and by consulting the original documents again. We provide a qualitative analysis of information obtained from methods papers to show the range of different approaches currently in place at the agencies in our sample and descriptive statistics for the analyses of HTA reports.

\section{RESULTS}

\section{Agencies Included in Our Sample}

A total of 121 agencies were potentially eligible and eighteen fulfilled all inclusion criteria (see Figure 1). The agencies included (see Table 1) are diverse with regard to their scope and their role within the respective health care systems.

\section{Methods Papers Describing Efforts to Include Unpublished Data}

Methods papers published by eligible agencies varied considerably regarding length (ranging from 9 to 294 pages), as well as currentness, with the oldest documents having been published in 2000, and the newest ones published as drafts in 2011 (for a complete list, see Supplementary Table 3, which can be viewed online at http://dx.doi.org/10.1017/S026646231300072X). Some of the agencies contacted informed us that the methods papers were being revised at the time of our research or no longer reflected current practice and more recent, internal guidance was being followed instead. However, in the absence of more up-to-date, publicly available documents, these methods papers were still included. The documents also reflect the scope of the respective agency, thus being comprehensive or addressing exclusively the evaluation of drugs or medical devices. Some of the documents are specific 
Table 1. Agencies Included in the Analysis and Approaches Regarding Requests to Industry

\begin{tabular}{llc}
\hline Abbreviation & \multicolumn{1}{c}{ Agency's name (country) } & Approach \\
\hline AHRQ & Agency for Healthcare Research and Quality (United States) & 1 \\
ASERNIP-S & Australian Safety and Efficacy Register of New Interventional Procedures - Surgical (Australia) & 3 \\
CADTH & Canadian Agency for Drugs and Technologies in Health (Canada) & 1 \\
CRD & Centre for Reviews and Dissemination (United Kingdom) & 2 \\
DACEHTA & Danish Centre for Health Technology Assessment (Denmark) & 3 \\
DERP & Drug Effectiveness Review Project (United States) & 1 \\
DIMDI & Deutsches Institut für Medizinische Dokumentation und Information (Germany) & 3 \\
G-BA & Gemeinsamer Bundesausschuss (Germany) & 3 \\
GÖG & Gesundheit Österreich GmbH (Austria) & 2 \\
HAS & Haute Autorité de Santé (France) & 3 \\
HVB & Hauptverband der österreichischen Sozialversicherungsträger (Austria) & 3 \\
IQWiG & Institut für Qualitït und Wirtschaftlichkeit im Gesundheitswesen (Germany) & 1 \\
KCE & Belgian Health Care Knowledge Centre (Belgium) & 1 \\
LBI & Ludwig Boltzmann Institut für Health Technology Assessment (Austria) & 2 \\
MSAC & Medical Services Advisory Committee (Australia) & 4 \\
NICE & National Institute for Health and Clinical Excellence (United Kingdom) & 1 \\
PHARMAC & Pharmaceutical Management Agency (New Zealand) & 4 \\
TLV & Tandvårds- och Läkemedelsförmånsverket (Sweden) & 1
\end{tabular}

Note. Explanation: $1=$ Manufacturers are routinely approached; $2=$ Manufacturer requests may be carried out at the discretion of the HTA authors; $3=$ Manufacturer requests are not explicitly mentioned in the methods paper; $4=$ Manufacturers (or others) file an application with their data for the product to be assessed.

The information reflects the content of the methods papers available to us at the time of our search. Note: HAS bases its assessments on a review of the literature and dossiers from pharmaceutical companies. The only methods papers that we were able to identify, however, did not reflect this current practice.

guidelines for the respective agencies (e.g., DERP, LBI [for agencies' full names, see Table 1]), while others have a broader target audience (e.g., DACEHTA, CRD). Where guidance for obtaining data was given separately for assessing effectiveness and adverse effects (e.g., CRD, AHRQ), we limited our analysis to the former.

Requests to Industry. We found that HTA agencies pursue different approaches concerning the question as to whether data are requested from industry in the course of HTA production. These can be grouped into four broad categories: (i) manufacturers are routinely approached as part of the search for relevant literature (seven agencies); (ii) manufacturer requests may be carried out at the discretion of the HTA authors (three agencies); (iii) manufacturer requests are not explicitly mentioned in the methods paper (six agencies); (iv) manufacturers (or others) file an application with their data for the product to be assessed (two agencies). Differences between agencies' approaches to a considerable extent also reflect the respective national legal context (e.g., when assessments are based on submissions by manufacturers instead of comprehensive searches carried out by HTA agencies) (see Table 1).
Five of the seven agencies that routinely make requests to manufacturers provide details of these processes in their methods papers; these are summarized in Table 2.

Conditions for accepting and including unpublished data in reports vary between agencies. The following conditions were addressed by at least on agency: completeness, adequate detail, non-confidentiality, and the timely submission of the data (see Table 3 for examples).

Few documents contain detailed guidance on how to report requests to industry: CRD provides an example for the documentation of a request, CADTH foresees requests to manufacturers as a standard part of the documentation of the literature search, DERP explicitly states that companies providing dossiers should be accounted for in the results section of the report, and IQWiG also states that requests to industry have to be documented in the reports. NICE publishes a list of consultees on its Web site. Several agencies (e.g., GÖG, KCE) state that the literature search has to be documented with enough detail to allow reproduction, without explicitly addressing requests to industry.

Equally, only a few methods papers in our sample provide explicit guidance on how to report previously unpublished data 
Kreis et al.

Table 2. Standardized Formats for Requests to Industry

AHRQ

Addressee:

Content of requests:

Process details:

DERP

Addressee:

Content of requests:

Process details:

IQWiG

Addressee:

Content of requests:

Process details:

NICE

Addressee:

Content of requests:

Process details:
Manufacturers of products under review (drugs, devices or other products for which a manufacturer can be identified)

Scientific Information Packets containing

- information on products available from the product label

- information on published and unpublished studies concerning the product

Requests are managed by the Scientific Resource Center for AHRQ on behalf of the Evidence-based Practice Centers (EPCS) "to ensure consistency in the way Scientific Information Packets (SIPS) are requested and to ensure transparency by eliminating contact between the EPC conducting the review and the manufacturers of products being reviewed".

Pharmaceutical companies that manufacture any drug included in an individual report

Dossiers containing

- published studies (a list of citations for all relevant published studies of drugs licensed by the respective company)

- unpublished studies (trials or observational designs; with information on, e.g., study identifier, design, indication, patient population description, inclusion \& exclusion criteria, treatment of interest and all comparisons, outcomes measured, results; sufficient amount of details on methods to allow assessment of study quality)

- unpublished, supplementary data for published clinical studies (e.g., additional details about study methods, additional outcomes, and results of additional subgroup analyses)

- a copy of the most recent product label

Requests are managed by the Medical Director for DERP, Center for Evidence-based Policy. As all information submitted may be made available to the public, all statements of confidentiality are considered "null and void".

Manufacturers of the technology under review

For drugs, a 2-step procedure is applied:

- 1) complete list of all trials carried out with the drug under assessment from the manufacturer

- 2) detailed request on the basis of 1) for unpublished studies or for additional, unpublished information on published studies Before submission of data, an agreement is made between IQWiG and the manufacturer involved specifying the submission process, the requirements for documents to be submitted (e.g., information provided should comply with CONSORT), and their confidential and non-confidential components. If a manufacturer does not agree to this contract (especially with regard to the complete transfer of all information requested) or does not completely transfer the information requested despite this contract, no further requests are made to this manufacturer.

Manufacturers and sponsors (organizations that market the technology under license) of the technology under review

Submissions, i.e., a "concise, comprehensive and structured report of all relevant information (published and unpublished) for an appraisal", containing

- a complete list of all studies on the technology within the indication (i.e., studies may be sponsored by manufacturers or sponsors or known to them; NICE may request further information on studies included in the list)

- a main document including, amongst other things, the aims of treatment, approved indications and an assessment of clinical effectiveness

- appendices containing supporting information for data and analyses and the excluded evidence

At the start of a project, manufacturers and sponsors are invited to take part in the appraisal as consultees. Consultees have at least 14 weeks to prepare their submissions which are then forwarded in full to the independent academic group preparing the evidence assessment report for NICE. in HTA reports: DERP provides complete copies of dossiers to the public upon request once the report is published. IQWiG foresees the possibility to publish short summaries of previously unpublished studies in its reports, as well as any additional relevant unpublished data. The methods papers of both agencies point out that all previously unpublished data included in the analyses are reported. If a submission to NICE contains previously unpublished confidential information, the manufacturer has to provide a second version with the confidential information removed, which is then published on the NICE Web site as a supplementary document together with the assessment. For previously unpublished trials, NICE requests a structured abstract for public disclosure. Information that has been submitted in confidence is removed from the actual HTA 
Table 2. Continued.

TLV

Addressee: $\quad$ Companies concerned by a pharmaceutical reimbursement review

Content of requests: $\quad$ Submission containing

- general information on the drug (e.g., administration form, dosage)

- information regarding the indication (e.g., average treatment time)

- information regarding medical effects: information on the "most important and well-executed studies" that have been conducted on the medicines (information for each of these studies in a table format, including primary and secondary effect measure, effect of treatment [intention to treat and per protocol analysis], losses to follow up etc.); summary of the medical effect of treatment based on these studies; summary of the effect of treatment on the patient's quality of life and life expectancy; advantages of the medicine regarding side effects compared to other medicines in this class of pharmaceuticals; known variations in the medical effect of the medicine (e.g., in respect to patients' sex and age)

- information regarding cost-effectiveness

Process details: When commencing the review of a therapeutic group, the companies responsible for marketing drugs in this group are notified by the TLV. In this context, they are requested to submit information on, amongst other things, the clinical use of and cost-effectiveness of the drug or drugs marketed by the company by filling in a standard form within 8 weeks.

Note. The information reflects the content of the methods papers that were available to us at the time of our search (see Supplementary Table 3 for a full list of references).

Table 3 Conditions for the Acceptance of Unpublished Data (Examples)

\section{Completeness:}

IQWiG has an agreement with manufacturers containing an obligation for complete submission of all relevant data. NICE requires manufacturers and sponsors to sign a statement declaring that all relevant material has been disclosed, and PHARMAC requests a declaration that all known unpublished clinical trials have been disclosed.

\section{Adequate detail:}

DERP requests sufficient amount of detail on methods of unpublished studies to allow for adequate assessment in order to include their data in the report. IQWiG requests companies to comply with the CONSORT statement when submitting information. PHARMAC allows abstracts and posters to be used by manufacturers in their applications only "as references to update information after the primary analyses or any analyses of secondary outcomes not detailed in the published report", if they are adequately detailed. Non-confidentiality:

DERP and IQWiG only accept data for inclusion in their analysis that may be made public; others (KCE, NICE, PHARMAC) also accept - under certain circumstances - data that are submitted in (either academic or commercial) confidence.

Timely submission of data:

DERP specifies that unpublished data or studies cannot be submitted by pharmaceutical companies after the deadline for the dossier process (e.g., not via the public commenting process for draft reports).

Note. The information reflects the content of the methods papers available to us at the time of our search (see Supplementary Table 3 for a full list of references).

report by blackening the relevant passage. Several agencies (e.g., PHARMAC, MSAC, TLV) point out that the respective legal requirements concerning the disclosure of confidential information apply.

Searches in Publicly Available Sources. Trial registries, regulatory authorities and conference abstracts all seem to play a similar role as additional sources: routine or optional searches in these sources are described in the methods papers of 9,11 , and 8 of eighteen agencies, respectively (see Supplementary Table 4, which can be viewed online at http://dx.doi.org/10.1017/S026646231300072X). Although the distinction between routine and optional parts of the literature search is not always clear cut, it seems that searching for conference abstracts is less often defined as a routine element. Table 4 depicts options for searching in these sources that we identified across agencies. 
Kreis et al.

Table 4. Options for Searching in Publicly Available Sources

Trial registries:

- Where: ClinicalTrials.gov (www.clinicaltrials.gov); Current Controlled trials (www.controlled-trials.com); WHO International Clinical Trials Registry Plafform

(www.who.int/trialsearch); Clinical Study Results (www.clinicalstudyresults.org) ${ }^{a}$ and further public or commercially owned registries

- To what end: identify unpublished studies; identify additional details of published studies; identify ongoing studies; contact principle investigators for further information

Regulatory authorities:

- Where: FDA (Medical and Statistical Reviews); EMA (European Public Assessment Reports) and further national authorities

- To what end: compare data from regulatory documents with results reported in respective publications to verify or find additional data; identify unpublished studies; compare

results from published and unpublished studies

Conference abstracts:

- Where: specialized databases; conference proceedings of selected meetings via hand searching

- To what end: identify studies that are not (yet) formally published in a peer-reviewed journal or indexed by electronic databases; compare preliminary data from abstracts with formally published results to check for possible inconsistencies or find additional data; contact abstract authors for full reports

Note. The information reflects the content of the methods papers available to us at the time of our search (see Supplementary Table 3 for a full list of references).

a: This registry which was set up by PhRMA is no longer available.

\section{HTA Reports Describing Efforts to Include Unpublished Data}

We identified seventy-three HTA reports published between 2006 and 2011 (thirty-three on drugs and forty on nondrug interventions [one report assessed both drug and nondrug interventions and was analyzed twice]) in our analysis. Nine of the HTA reports in our sample assessed nondrug interventions that do not involve the application of a medical device (for example, psychosocial interventions for people bereaved by suicide); we thus excluded them from further analyses regarding requests to industry and searches of regulatory authority Web sites.

Requests to Industry. Current practices described in HTA reports broadly reflect the methods outlined in their methods papers: of those agencies with routine requests (see above), twenty-one of thirty-one eligible reports ( 68 percent) explicitly mentioned that requests to industry were carried out. While it remained unclear in three reports whether any response was received, eighteen reports described the receipt of information, although in some cases not all of the manufacturers contacted responded. Half of these responses $(n=9)$ apparently yielded data previously not publicly accessible; one explicitly stated that no additional information had been retrieved, and for eight reports it was not clearly described.

Of those agencies where requests to industry are at the discretion of the HTA authors, only four of nine reports (44 percent) described a corresponding request; in all cases it remains unclear whether a response was received. None of the seventeen reports of agencies where requests to industry were not mentioned in the methods paper described a corresponding request.

An analysis of the extent to which previously unpublished data were included in the statistical analyses and their possible impact on results turned out to be unfeasible in view of this sparse reporting.

Seven reports were included of those agencies where manufacturers (or others) file an application for the product to be assessed (HAS, MSAC, PHARMAC). While three reports of MSAC did not include unpublished data (possibly reflecting the fact that these reports are carried out separately from the assessment of the manufacturer's application), two of the three HAS reports included unpublished data. Data submitted to PHARMAC included slides presented at conferences and further data submitted in confidence.

Searches in Publicly Available Sources. In thirty-eight of seventy-three reports (52 percent) in our sample, trial registries were searched. Most reports stated that this was done to identify ongoing trials, but some also described that this served to identify both ongoing and unpublished trials. The registry most often searched was ClinicalTrials.gov. In twenty-five of sixty-four reports (39 percent) involving the use of drugs or medical devices, regulatory authority Web sites (mostly the FDA) were searched to identify further documents. In twelve of seventy-three reports (16 percent), the literature search explicitly encompassed hand searching of selected conference proceedings. Of the remaining reports, many reported searches in specialized electronic databases, for example, Conference Papers Index, Inside Conferences or ISI Proceedings.

\section{DISCUSSION}

\section{Policies and Practices Regarding Requests to Industry}

Although the neglect of unpublished data can seriously threaten the validity of HTA reports, international HTA agencies currently differ considerably in their efforts to address this issue, 
both with regard to the question as to whether and how industry is asked for unpublished data and to policies concerning the reporting and publishing of data received. Current practices vary according to the different policies implemented by the agencies, where only a minority of agencies foresee routine data requests to industry. Surprisingly, in those reports where manufacturers were approached, the reporting of the request and of the data retrieved was often sparse. It, therefore, often remained unclear whether and which manufacturers were approached, whether they provided data, and whether or where these were included in the statistical analysis.

\section{Alternative Strategies to Identify Unpublished Data}

In approximately half of the reports, the search in bibliographic databases was supplemented by a search in trial registries, although it was often not clearly stated whether the main intent of the search was to identify ongoing studies or studies not (yet) published. Some HTA authors expended considerable effort in identifying unpublished randomized controlled trials from trial registries (e.g., $(18 ; 19))$.

The handling of conference abstracts varied across agencies. Abstract publications pose a serious problem for HTA authors, as their inclusion may be important to reduce the influence of reporting bias, yet they may lack important information (20). While many reports excluded abstracts, some HTA authors decided to include studies on the basis of abstracts alone to ensure that as much evidence as possible was included, as long as enough information for appraising the study's quality was available (e.g., (19;21)). It is noteworthy in this context that the methods papers of the HTA agencies often did not specify how to proceed with conference abstracts once identified.

Although this was not the focus of our research, several reports in our sample explicitly discussed the problem of publication bias or missing data (e.g., (22-25)), and some applied strategies such as funnel plots or Egger's weighted regression statistics to investigate possible publication bias (e.g., $(25 ; 26)$ ). While many reports put considerable effort into trying to identify unpublished data by means of different strategies, some HTA authors decided as a matter of principle not to search for unpublished data to maintain the reproducibility of the data included (e.g., (27)), or because unpublished data are difficult to search for systematically and exhaustively (e.g., (28)).

\section{POLICY IMPLICATIONS}

As noted above, we did not assess the impact of requests to industry for unpublished data on the conclusions of the HTA reports as the current extent and quality of information did not allow for such an analysis. The actual impact of requests to industry therefore remains unclear for the reports in our sample. However, as long as legislation does not require all trials to be registered and the reporting of results in registries is incomplete (29), there are strong arguments for such requests.
For those agencies or research groups that consider carrying out requests to industry in the future, some lessons may be learned from those agencies that already routinely do so. As some methods papers highlight, it is important to ensure that all relevant unpublished studies are disclosed upon request (e.g., by requesting corresponding declarations from manufacturers): if data were provided selectively, this would possibly even increase reporting bias. Standardized request forms following CONSORT guidelines (30) are a way to facilitate requests to prevent selective reporting; ideally, full study reports should be made available upon request. Efforts should also be made to make data provided by manufacturers publicly available as soon as possible to ensure that assessments are transparent and the ensuing decisions open to scrutiny (31).

Given the sparse reporting of requests to industry found in many of the reports assessed, it would be desirable to develop reporting standards for such requests and for the information they yield. Although reporting guidelines for systematic reviews require all data sources to be stated (32), detailed guidance for reporting requests to industry is currently lacking. We suggest that these should include the manufacturers approached, whether they provided data and the publication status of these data (see also (33)).

However, some reports in our sample (e.g., (23;34)), as well as further examples such as the reboxetine case (5) or the Cochrane Review on Tamiflu (35), show that routine inquiries alone are often insufficient, and despite considerable persistence and investment of resources, all relevant data may not always be provided, as in the case of Tamiflu $(6 ; 36)$.

Ideally, requests to industry will be made superfluous in the future by legislative measures that guarantee HTA agencies access to relevant data, as already implemented for early benefit assessments of drugs in Germany (37). A further groundbreaking initiative in the area of drug assessments is EMA's new policy of releasing marketing authorization documents on request (e.g., clinical study reports), after the decision-making process for the application of the drug in question is finalized (38). This policy has been legally challenged: in spring 2013, the General Court, as a result of complaints filed by two pharmaceutical companies, issued a temporary injunction ordering EMA not to provide any documents until a final ruling is given (39). Despite this, EMA is planning proactive publication of such information depending on the type of data (40). For nondrug interventions, however, the road to full availability of relevant data seems to be much longer. Initiatives to promote the prospective public registration of studies and disclosure of data (e.g., (41)) may, therefore, remain more important for studies evaluating medical devices or other nondrug interventions.

\section{Strengths and Weaknesses of Our Study}

We aimed to create a comprehensive and systematic approach for identifying HTA agencies to obtain an unbiased picture of the HTA community. The analysis of both methods papers 
and recent HTA reports allowed capturing not only the policies specified by the agencies, but also actual practices.

Challenges we encountered were the considerable intertwining of agencies in their work (i.e., mutual commissioning of reports or mutual use of methods papers), the fact that several HTA agencies produce different types of documents, and difficulties in always clearly distinguishing between "real" HTA reports and assessments triggered by manufacturer applications.

Our study has some limitations. Approximately a quarter of nondrug interventions assessed in the HTA reports of our sample did not involve the application of a medical device so that manufacturers could not be contacted. We did not systematically evaluate whether in these cases other possible owners of data, e.g., research groups at universities, were contacted. Selective publication of studies, however, may be equally (or even more) prevalent in academic research, especially with regard to study designs other than clinical trials. A challenge in the evaluation of interventions not commercially owned is that it is less clear who may have carried out studies or who may be in possession of the data, and the diversity of relevant parties in this field may make requests difficult.

In addition, our analysis, which was exploratory in nature, was restricted to information available in publicly available documents, and, despite our efforts, we may have failed to identify all relevant documents. Some methods papers may also not reflect current policies, as indicated by some of the respondents to our inquiries. In the absence of other information available, we treated methods papers equally, regardless of considerable differences with regard to their comprehensiveness and their currentness. As the consideration of reporting bias in HTA has changed over time, differences between methods papers in this regard may also be related to the time at which they were written. Inevitably, our study does not reflect current activities or discussions at the HTA agencies that are yet unconsidered in official documents. Thus, future research could strive for a more extensive participatory approach to expand our knowledge on strategies identified in our study and agencies' experiences.

\section{CONCLUSIONS}

It seems self-evident that HTA reports influencing health care decisions for millions of patients should be based on all relevant data. To different extents, HTA agencies search trial registries, regulatory authorities' public documents or conference abstracts to identify studies and data not (yet) published in peerreviewed journals. In future, HTA agencies should be guaranteed comprehensive access to relevant data either by compulsory registration of study protocols and comprehensive results in public trial registries or by being granted access to clinical study documents submitted to regulatory authorities. Until then, requests to industry may represent one strategy to access and include unpublished data, while agencies can learn from each other concerning successful practice. Furthermore, the devel- opment of reporting standards for such requests and for data obtained from manufacturers would help to improve the transparency of HTA reports.

\section{SUPPLEMENTARY MATERIAL}

Supplementary Table 1-4: http://dx.doi.org/10.1017/

S026646231300072X

\section{CONTACT INFORMATION}

Julia Kreis, Dipl.-Psych., (julia.kreis@iqwig.de), Research Associate, Institute for Quality and Efficiency in Health Care (IQWiG), Cologne, Germany

Dimitra Panteli, MD, MscPH, Research Fellow, Department of Health Care Management, University of Technology, Berlin, Germany

Reinhard Busse, MD, MPH, FFPH, Professor and Department Head, Department of Health Care Management, University of Technology, Berlin, Germany

\section{CONFLICTS OF INTEREST}

Reinhard Busse has received travel support from the European Observatory on Health Systems and Policies. Julia Kreis is an employee of IQWiG. In order to produce unbiased health technology assessment reports, the institute depends on access to all of the relevant data on the topic under investigation. Kreis therefore supports the mandatory worldwide establishment of trial registries and public access to clinical study reports. The other authors report they have no potential conflicts of interest.

\section{REFERENCES}

1. Sterne JAC, Egger M, Moher D, on behalf of the Cochrane Bias Methods Group. Addressing reporting biases. In: Higgins JPT, Green S, eds. Cochrane handbook for systematic reviews of interventions. Chichester, UK: Cochrane Collaboration and John Wiley \& Sons; 2008.

2. McGauran N, Wieseler B, Kreis J, et al. Reporting bias in medical research - A narrative review. Trials. 2010;11:37.

3. Higgins JPT, Altman DG, on behalf of the Cochrane Statistical Methods Group and the Cochrane Bias Methods Group. Assessing risk of bias in included studies. In: Higgins JPT, Green S, eds. Cochrane handbook for systematic reviews of interventions. Chichester, UK: Cochrane Collaboration and John Wiley \& Sons; 2008.

4. Song F, Parekh S, Hooper L, et al. Dissemination and publication of research findings: An updated review of related biases. Health Technol Assess. 2010;14:iii, ix-xi, 1-193.

5. Eyding D, Lelgemann M, Grouven U, et al. Reboxetine for acute treatment of major depression: Systematic review and meta-analysis of published and unpublished placebo and selective serotonin reuptake inhibitor controlled trials. BMJ. 2010;341:c4737.

6. Godlee F, Loder E. Missing clinical trial data: Setting the record straight. BMJ. 2010;341:c5641.

7. Code of Federal Regulations. Title 21 - Food and Drugs Chapter - Food and Drug Administration of Health and Human Services. Subchapter D - Drugs for Human Use. Part 314 - Applications for FDA Approval to Market a New Drug. Subpart B-ApplicationsSec. 314.50 Content and format of an application (21CFR314.50). 
8. Wieseler B, McGauran N, Kaiser T. Finding studies on reboxetine: A tale of hide and seek. BMJ. 2010;341:c4942.

9. Laurance J. Drug giants warned: Tell the truth on medicines. The Independent. 27.02.2008.

10. Depressing research. Lancet. 2004;363:1335.

11. De Angelis C, Drazen JM, Frizelle FA, et al. Clinical trial registration: A statement from the International Committee of Medical Journal Editors. Lancet. 2004;364:911-912.

12. New York State Attorney General. Settlement sets new standard for release of drug information [online]. http://www.ag.ny.gov/pressrelease/settlement-sets-new-standard-release-drug-information (accessed December 2, 2012).

13. Wood AJ. Progress and deficiencies in the registration of clinical trials. $N$ Engl J Med. 2009;360:824-830.

14. European Medicines Agency. About EU Clinical Trials Register [online]. https://www.clinicaltrialsregister.eu/about.html (accessed August 29, 2013).

15. Turner EH. Closing a loophole in the FDA Amendments Act. Science. 2008;322:44-46.

16. Lefebvre C, Manheimer E, Glanville J, on behalf of the Cochrane Information Retrieval Methods. Searching for studies. In: Higgins JPT, Green $\mathrm{S}$, eds. Cochrane handbook for systematic reviews of interventions. Chichester, UK: Cochrane Collaboration and John Wiley \& Sons; 2008.

17. Smith R. What is publication? BMJ. 1999;318:142.

18. Yank V, Tuohy CV, Logan AC, et al. Comparative effectiveness of inhospital use of recombinant factor VIIa for off-label indications vs. usual care. Rockville (MD): Agency for Healthcare Research and Quality (US); 2010 May. Report No.: 10-EHC030-EF.

19. Fayter D, Corbett M, Heirs M, Fox D, Eastwood A. A systematic review of photodynamic therapy in the treatment of pre-cancerous skin conditions, Barrett's oesophagus and cancers of the biliary tract, brain, head and neck, lung, oesophagus and skin. Health Technol Assess. 2010;14:1-288.

20. Hopewell S, McDonald S, Clarke M, Egger M. Grey literature in metaanalyses of randomized trials of health care interventions. Cochrane Database Syst Rev. 2007;2:MR000010.

21. Smith B, Carson S, Fu R, et al. Drug class review: Disease-modifying drugs for multiple sclerosis. Final update 1 report. Portland (OR): 2010. Oregon Health \& Science University; 2010 Aug.

22. Wessling A, Ramsberg J. The review of antidepressants. Solna: TLV; 2008.

23. Metcalfe S, Burgess C, Laking G, et al. Trastuzumab: Possible publication bias. Lancet. 2008;371:1646-1648.

24. Buchberger B, Follman M, Freyer D, et al. Bedeutung von Wachstumsfaktoren für die Behandlung von chronischen Wunden am Beispiel des diabetischen Fußulcus. Köln: DAHTA; 2010. (Schriftenreihe Health Technology Assessment).

25. Coleman CI, Baker WL, Kluger J, et al. Comparative effectiveness of angiotensin converting enzyme inhibitors or angiotensin II receptor blockers added to standard medical therapy for treating stable ischemic heart disease. Rockville (MD): 2009. Agency for Healthcare Research and Quality (US); 2009 Oct.

26. Neyt M, Van den Bruel A, Gailly J, Thiry N, Devriese S. Tiotropium in the treatment of chronic obstructive pulmonary disease: Health technology assessment. KCE reports 108C. Brussels: Bel- gian Health Care Knowledge Centre (KCE); 2009. (Health Technology Assessment).

27. Gorenoi V, Schönermark MP, Hagen A. Infektionsschutz in der Knieendoprothetik. Köln: DAHTA; 2010. (Schriftenreihe Health Technology Assessment).

28. Medical Services Advisory Committee. Cryotherapy for recurrent prostate cancer and renal cancer: Part A - Salvage cryotherapy for recurrent or persistent prostate cancer after radiotherapy. 2009. (MSAC application 1124).

29. Prayle AP, Hurley MN, Smyth AR. Compliance with mandatory reporting of clinical trial results on ClinicalTrials.gov: Cross sectional study. BMJ. 2012;344:d7373.

30. Moher D, Hopewell S, Schulz KF, et al. CONSORT 2010: Explanation and elaboration; Updated guidelines for reporting parallel group randomised trials. BMJ. 2010;340:c869.

31. Drummond MF, Schwartz JS, Jonsson B, et al. Key principles for the improved conduct of health technology assessments for resource allocation decisions. Int J Technol Assess Health Care. 2008;24:244-258.

32. Moher D, Liberati A, Tetzlaff J, Altman DG, Group P. Preferred reporting items for systematic reviews and meta-analyses: The PRISMA statement. Ann Intern Med. 2009;151:264-269, W264.

33. Niederstadt C, Droste S. Reporting and presenting information retrieval processes: The need for optimizing common practice in health technology assessment. Int J Technol Assess Health Care. 2010;26:450457.

34. Institut für Qualität und Wirtschaftlichkeit im Gesundheitswesen. Selektive Serotonin- und Noradrenalin-Wiederaufnahmehemmer (SNRI) bei Patienten mit Depressionen: Abschlussbericht; Auftrag A0520A; Version 1.1 [online]. 18.08.2010 (IQWiG-Berichte; Band 55). http://www.iqwig.de/download/A05-20A_Abschlussbericht_SNRI_bei_ Patienten_mit_Depressionen_V1-1.pdf (accessed August 29, 2013).

35. Jefferson T, Jones MA, Doshi $P$, et al. Neuraminidase inhibitors for preventing and treating influenza in healthy adults and children. Cochrane Database Syst Rev. 2012;1:CD008965.

36. Payne D. Tamiflu: The battle for secret drug data. BMJ. 2012;345: e7303.

37. Gesetz zur Neurordnung des Arzneimittelmarktes in der gesetzlichen Krankenversicherung (Arzneimittelmarktneuordnungsgesetz - AMNOG) vom 22. Dezember 2010. Bundesgesetzblatt Jahrgang. 2010 Teil I Nr. 67.

38. European Medicines Agency. European Medicines Agency widens public access to documents [online]. 30.11.2010. http://www.ema.europa.eu/ docs/en_GB/document_library/Press_release/2010/11/

WC500099468.pdf (accessed August 29, 2013).

39. European Medicines Agency. European Medicines Agency receives interim decisions of the General Court of the EU on access to clinical and non-clinical information [online]. 30.04.2013. http://www.ema. europa.eu/docs/en_GB/document_library/Press_release/2013/04/ WC500142837.pdf (accessed August 29, 2013).

40. European Medicines Agency. Publication and access to clinicaltrial data [online]. 24.06.2013. http://www.ema.europa.eu/docs/en_GB/ document_library/Other/2013/06/WC500144730.pdf (accessed August 29, 2013).

41. Godlee F. Clinical trial data for all drugs in current use. BMJ. 2012;345:e7304. 